\title{
Hydrophobic properties of the cell surface of Candida albicans: a role in aggregation
}

\author{
Carole Hobden, Claire Teevan, Lorraine Jones and Paul O'Shea
}

Author for correspondence: Paul O’Shea. Tel: +44 1206 872043. Fax: +44 1206873598.
e-mail: poshea@, essex.ac.uk

Department of Chemistry and Biological Chemistry, The University of Essex, Wivenhoe Park, Colchester, Essex CO4 3SQ, UK

\begin{abstract}
The ability of Candida albicans to aggregate and adhere to biological surfaces is a topic of major biological and medical importance. One factor which has been implicated in such properties is the hydrophobic nature of the cell surface. Two simple spectroscopic techniques are described which permit the rapid determination of this property. The first involves the use of aryinaphthalenesulfonate, the fluorescence emission maximum of which was shown to be a sensitive indicator of dielectric polarity. This was used to identify the hydrophobic characteristics of the cell surface of $C$. albicans. The second technique involves the use of $90^{\circ}$ Rayleigh-Debye light scattering as an indicator of the aggregation state of a fungal suspension. These techniques were then used to compare the surface properties of three different strains of C. albicans and the effects of culture conditions: the hydrophobicity of the strains varied, and galactose-based culture media promoted the greatest degree of cell surface hydrophobicity.
\end{abstract}

Keywords: Candida albicans, dielectric constant, light scattering, surface charge, adhesion

\section{INTRODUCTION}

There is much interest in the properties of the cell surface of opportunistic fungal pathogens such as Candida albicans, because this structure must be the site of contact and thus the locus of infection of prospective hosts. The adherence of $C$. albicans to buccal and vaginal epithelial cells, for example, is necessary for the establishment of oral and vaginal thrush, respectively (Hazen, 1989; Reinhart $e$ t al., 1985). Similarly, adhesion must also occur to host cells before the appearance of the well-defined pathogenic sequelae of systemic infections in immunocompromised hosts (Meunier, 1989). Possibly in a related manner, $C$. albicans also tends to self-aggregate, which results in some protection from therapeutic intervention (Calderone \& Braun, 1991); and finally, these fungi may colonize prosthetic devices and catheters (Rotrosen et al., 1983). The fungal cell wall/surface, therefore, would appear to be involved in all these processes, exhibiting both specific and non-specific properties of molecular recognition with themselves (Jones \& O'Shea, 1994), host structures (Hazen, 1989; Calderone \& Braun, 1991) and artificial surfaces (Hazen, 1990; Jones \& O'Shea, 1994). Work in

Abbreviations: ANS, 8-anilino-1-naphthalene sulfonic acid; CSH, cellsurface hydrophobicity; $\varepsilon$, dielectric constant; $\varepsilon_{s,}$ scattering coefficient; $I_{s,}$ scattering intensity. our laboratory has shown that $C$. albicans appears to possess two properties that influence this behaviour. First, the cell surface appears to be negatively charged and consequently possesses an electronegative potential (O'Shea, 1991; Jones \& O'Shea, 1994). Of these two related parameters, the latter is the most relevant in thermodynamic terms and, therefore, the most influential concerning possible interactions (presumably nonspecific) with other surfaces both biological and manmade. The chemical groups that confer the negative charges may also be involved in more specific chargecharge interactions with mammalian cells. It has been reported, for example, that sialic acid is present on the cell surface of $C$. albicans, and it was postulated that this may play a role in specific cell recognition processes as well as contributing to the overall surface electrostatic potential (Jones et al., 1995).

Secondly, the cell surface of $C$. albicans appears to possess a certain degree of hydrophobicity, and this may be involved in fungal adhesion (Douglas, 1985; Klotz \& Penn, 1987; Hazen, 1990). Furthermore, the hydrophobic properties of the cell surface of $C$. albicans appear to exhibit significant variation depending on the strain as well as the growth medium and conditions (Hazen et al., 1986; McCourtie \& Douglas, 1981, 1984). C. albicans has also been reported to produce surfactants which make the cell surface more hydrophobic. These surfactants may be 
cell-wall-associated or released into the extracellular surroundings (Kaeppeli et al., 1978; Klotz \& Penn, 1987). The cell-associated surfactant appears to contribute to the ability of $C$. albicans to bind to epithelial cells (Klotz \& Penn, 1987) and to inert surfaces (Klotz, 1989). Adhesion of Candida species to some epithelial cells appears to involve a number of mechanisms. The underlying mechanism of attraction may arise from the hydrophobic effect (Klotz \& Penn, 1987; Hazen, 1989). A second possibility relates to the putative existence of an adhesin-receptor interaction and the identity of the adhesin has been linked with a mannoprotein (McCourtie \& Douglas, 1984; Critchley \& Douglas, 1987).

It is well documented that the cell surface of $C$. albicans has distinct areas which are either charged (Jones \& O'Shea, 1994; Jones et al., 1995) or hydrophobic in nature (Kennedy, 1990; Hazen, 1989). The electrostatic interactions between cell surfaces are thought solely to be repulsive under the majority of circumstances and actively oppose adhesion. The second physical property of $C$. albicans, i.e. the cell-surface hydrophobicity (CSH), however, can only promote adhesion. The balance between these attractive and repulsive forces, therefore, would appear to determine whether or not adhesion or aggregation may occur for the population of cells as a whole.

It is thought that CSH is determined by many intrinsic and extrinsic factors and this is reminiscent of similar studies with bacteria (Rosenberg \& Kjelleberg, 1986). The extent of CSH of $C$. albicans is temperature-dependent (Hazen \& Hazen, 1987; Kennedy \& Sandin, 1988); C. albicans isolates generally appear more hydrophobic when grown to stationary phase at room temperature $\left(23-25^{\circ} \mathrm{C}\right)$ than at $37^{\circ} \mathrm{C}$. The nature of the growth medium is also known to influence the extent of $\mathrm{CSH}$ for $C$. albicans (Hazen et al., 1986; Kennedy \& Sandin, 1988; Hazen, 1990; Kennedy, 1990).

The determination of the CSH of fungi, and $C$. albicans in particular, remains a matter of contention. Klotz et al. (1985) and Minagi et al. (1986), for example, have used the so-called 'contact angle' technique to determine CSH. Minagi et al. (1986) have also used a microbial adhesion to hydrocarbons (MATH) assay to characterize the CSH of $C$. albicans. There are a number of other such methods (Smyth et al., 1978; Rosenberg et al., 1980; Lindahl et al., 1981; Lachica \& Zink, 1984), but none have been used to study $C$. albicans. All of these techniques have inherent technical difficulties. In particular, the contact angle technique is fraught with difficulty and technical problems [some of which are outlined in a review of CSH by Hazen (1990)], such as the purity of the 'contact' solvent in the presence of fungi. The question of the surface disposition of the hydrophobic moieties may also seriously complicate the interpretation.

In the present study, the extent of $\mathrm{CSH}$ as a function of the strain and growth conditions has been investigated using two complementary techniques. The first involves a fluorescent probe, 8-anilino-1-naphthalene sulfonic acid (ANS), whose emission spectrum is highly sensitive to dielectric polarity (Slavik, 1982; Hobden et al., 1995) and may therefore offer the means to determine the $\mathrm{CSH}$ of $C$. albicans. The second technique employs a novel application of light scattering for indicating the degree of aggregation of the $C$. albicans, which is presumably also influenced by $\mathrm{CSH}$.

\section{METHODS}

Strains and growth conditions. C. albicans strain MRL 3153 was supplied as a freeze-dried culture from the British Mycological Reference Laboratory, Collingdale, London, UK. The culture was revived with $0.5 \mathrm{ml}$ liquid medium added to the ampoule containing the culture, mixed and left for a few minutes. The suspension was then used to inoculate agar slopes. Strains GRI 681 and GDH 2346 were supplied on agar slopes by SmithKline Beecham. The three strains of yeast were subcultured from the agar slopes and independently cultured in three different media: RPMI 1640 (1 M glucose, Sigma), Yeast Nitrogen Base (YNB, Sigma) supplemented with $500 \mathrm{mM}$ galactose (YNB + Gal), and YNB supplemented with $50 \mathrm{mM}$ glucose (YNB+Glc). The fungi were incubated at $37^{\circ} \mathrm{C}$ and harvested during exponential growth. The yeast cells were centrifuged at $2000 \mathrm{~g}$ for $5 \mathrm{~min}$, the supernatant was aspirated and discarded and the cells were resuspended in $1 \mathrm{mM}$ HEPES, $\mathrm{pH} 7 \cdot 5$. This procedure was repeated three times. The final concentration of cells was adjusted to $\sim 6 \times 10^{6}$ cells $\mathrm{ml}^{-1}$. Microscopic examination of the cells at this stage indicated that the $C$. albicans appeared to be predominantly yeast rather than hyphal.

Spectrophotometry. All spectrofluorimetric measurements were done with a Perkin-Elmer LC-50 spectrofluorimeter linked to a microcomputer running under MS-DOS. Data were stored in binary and ASCII file formats and presented with appropriate graphics and data-fitting software (Biosoft). Following excitation of ANS at $360 \mathrm{~nm}$, emission spectra were recorded over the range $420-570 \mathrm{~nm}$ with solutions made up in the solvents with dielectric constants (relative permittivities) $(\varepsilon)$ ranging from 2 to 80 (Table 1). Studies with 1-5 mM ANS in each solvent were done, the concentration used depending upon the fluorescence yield.

To study the interaction of ANS with $C$. albicans, aqueous suspensions of fungi were mixed with $10 \mu \mathrm{M}$ ANS and emission spectra were recorded following excitation at $360 \mathrm{~nm}$. The ANS stock solution was prepared in ethanol; the final concentration of ethanol present with the cell suspensions was never greater than $0.2 \%$. The emission maximum $\left(\lambda_{\max }\right)$ for each $C$. albicans preparation was then recorded. From the relationship between

Table 1. Dielectric constants of solvents used to study the fluorescence of ANS

\begin{tabular}{|lc|}
\hline Solvent & $\begin{array}{c}\text { Dielectric } \\
\text { constant }(\varepsilon)\end{array}$ \\
\hline Carbon tetrachloride & 2 \\
Hexanol & 13 \\
Propanol & 20 \\
Ethanol & 24 \\
Methanol & 32 \\
Ethylene glycol & 41 \\
Water & 80 \\
\hline
\end{tabular}


$\lambda_{\max }$ and $\varepsilon$ (see Fig. 1), it is possible to identify an 'equivalent' $\varepsilon$ $(\equiv \varepsilon)$ for the $C$. albicans cell surface as listed in Table 2.

Rayleigh-Debye scattering measurements were done with a Perkin-Elmer LC-50 spectrofluorimeter, by irradiating a filtered solution of $1 \mathrm{mM}$ HEPES, pH 7.5, at $600 \mathrm{~nm}$ and the intensity of the scattered light at $600 \mathrm{~nm}$ was recorded at $90^{\circ}$ to the incident radiation. Changes of the scattering intensity following the serial additions of aliquots of $5 \times 10^{5} \mathrm{C}$. albicans cells suspended in $1 \mathrm{mM}$ HEPES, $\mathrm{pH} 7 \cdot 5$, were then recorded.

Use of $90^{\circ}$ Rayleigh-Debye light scattering to assess the aggregation state of $C$. albicans: theoretical background. Classical scattering theory has been directed towards colloidal particles of biological origin for a number of years but as far as we are aware, has not hitherto been discussed in terms of the light-scattering properties of yeast cells. It is clear, however, that if the concentration of yeast cells in an aqueous suspension is increased then the suspension becomes more turbid. The study of such behaviour, known as turbidimetry (see, e.g. Latimer, 1979) is analogous to microscopy except that in the former the image is not reconstructed to form an image (i.e. inverse Fourier transform). Turbidimetry may instead yield a quantity known as the scattering coefficient $\left(\varepsilon_{\mathrm{s}}\right)$, which may be an absolute quantity, i.e. a constant for a given wavelength in the same manner as the absorption coefficient of the BeerLambert law. Thus, although the light transmitted through an increasingly turbid sample may be attenuated, the scattered light at certain angles increases proportionally. For the process of classical Rayleigh-Debye scattering, the following equation (1), quoted by Kerker (1969), is appropriate:

$\varepsilon_{\mathrm{s}}=\frac{9 \pi^{3} V^{2}}{\lambda^{4} \ln 10}\left(\frac{m^{2}-1}{m^{2}+2}\right)^{2} \int_{0}^{\pi} P(\theta)\left(1+\cos ^{2} \theta\right) \sin 0 \mathrm{~d} \theta$

where $V$ is the effective scattering volume of the particle, $P(\theta)$ is the intra-particle interference at a given observation angle $(\theta)$, $\lambda$ is the wavelength of light in the medium and $m$ is the refractive index. For essentially identical spherical particles such as those represented by yeast cells, and provided variables such as the wavelength of the incident radiation $(\lambda)$ and the scattering angle $(\theta)$ are held constant, equation (1) can be incorporated into the following expression, which relates the positive proportionality constant (equivalent to the scattering coefficient, $\varepsilon_{\mathrm{s}}$ ) to the cell concentration $(c)$ and the scattering intensity $\left(I_{\mathrm{s}}\right)$ :

$I_{\mathrm{s}}=\varepsilon_{\mathrm{s}} \cdot c$

The value of the $\varepsilon_{\mathrm{s}}$ for yeast cells may be obtained in a manner analogous to that of the Beer-Lambert law, i.e. by measuring the scattered light as a result of the increase of the concentration of the cell suspension. If the volume of the elementary particle (V) changes, however, this must also change $\varepsilon_{\mathrm{s}}$ and lead to a different proportionality between the scattering intensity and the cell concentration. This relationship is not quite as simple as implied because other shape factors also become significant. Nevertheless, by exploiting changes of the value of $\varepsilon_{\mathrm{s}}$ which occurs under a number of circumstances, the effective size of the 'unit yeast' particle can be determined. It is envisaged that the particle size represents that of the single cell for a dilute suspension of non-aggregating yeasts. A smaller value of $\varepsilon_{\mathrm{s}}$ might be anticinated, however, for a population of yeasts which are 'slightly' aggregated, e.g. consisting of aggregates of several particles. Such aggregates will exhibit an average number of single yeasts per aggregated particle which will determine the scattering properties of the population. For the purpose of this study, a relative value of $\varepsilon_{\mathrm{s}}$, as the gradient of $I_{\mathrm{s}}$ per unit concentration of yeast may be used to determine the aggregation state of the yeast population (Fig. 3). The gradient thus determined provides a simple means by which the ability of the yeasts to aggregate can be assessed (Table 2).

\section{RESULTS}

\section{Emission spectrum of ANS as an indicator of dielectric constant}

The emission spectrum of ANS was found to be highly sensitive to the dielectric constant of the solvent and a plot of this relationship was virtually linear (Fig. 1). The relationship between the intensity at $\lambda_{\max }$ (also shown in Fig. 1), however, was highly non-linear. There was an initial increase of fluorescence intensity as the solvent dielectric constant was increased, with a maximum at $\varepsilon$ 13-20. The intensity then fell rapidly with increasing $\varepsilon$, until it reached its lowest value at $\varepsilon 80$. A more detailed account of the spectroscopy of ANS may be found in Hobden et al. (1995).

\section{CSH of C. albicans as revealed by ANS spectrofluorimetry}

The emission spectrum of ANS in water and ANS supplemented with yeast cells of $C$. albicans are shown in Fig. 2. The emission spectrum of ANS in water exhibits a $\lambda_{\max }$ at $513 \mathrm{~nm}$ but is only weakly fluorescent (Fig. 1). ANS in the presence of $C$. albicans suspended in an aqueous solution promotes a spectral blue-shift as well as a large increase in the intensity. According to the relationship between the $\lambda_{\max }$ and $\varepsilon$, as shown in Fig. 1, some molecules of ANS must have moved from the highly polar environment $(\varepsilon=80)$ offered by water to an environment which is more hydrophobic $(\varepsilon=2-4)$, presumably the $C$. albicans cell surface. This spectral change occurred immediately following the addition of an aqueous solution of ANS to the $C$. albicans suspension and remained stable with no additional (i.e. time-dependent)

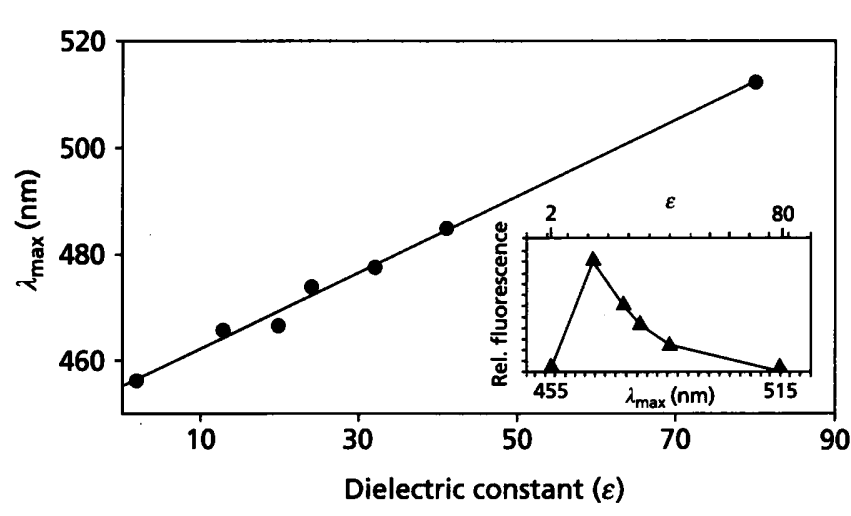

Fig. 1. Relationship between emission maxima of ANS and solvent polarity. ANS solutions were excited at $360 \mathrm{~nm}$ and the emission was measured at $420-570 \mathrm{~nm}$. The emission maxima $\left(\lambda_{\max }\right)$ and the fluorescence intensity at $\lambda_{\max }$ were noted and plotted as indicated. The inset shows the relationship between relative fluorescence intensity and $\lambda_{\text {max }}$. The $y$-axis scale is about $0-3000$ fluorescence units; on this scale the relative fluorescence of ANS at 455 and $513 \mathrm{~nm}$ is $\sim$ 5-10 units. 


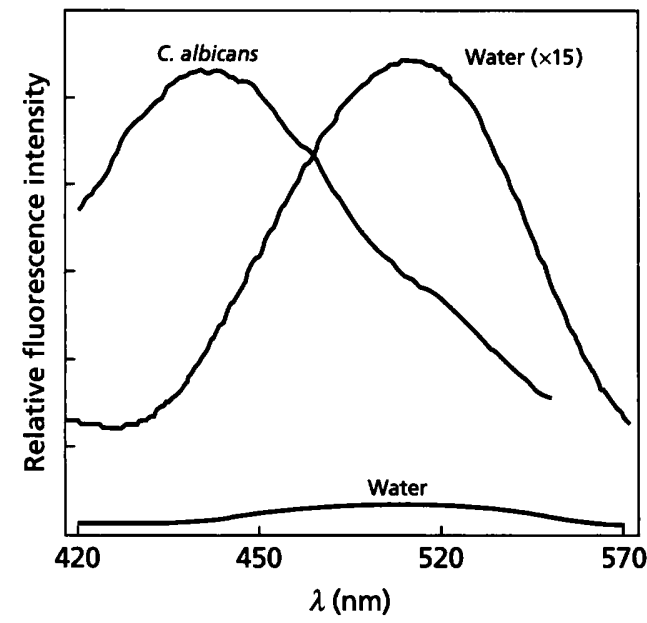

Fig. 2. Emission spectra of ANS in water and in the presence of C. albicans. Aqueous solutions of ANS $(10 \mu \mathrm{M})$ in the absence and the presence of $C$. albicans $\left(6 \times 10^{6}\right.$ cells $\left.\mathrm{ml}^{-1}\right)$ were excited at $360 \mathrm{~nm}$ and the emission was measured at $420-570 \mathrm{~nm}$. To facilitate comparison, the weakly fluorescent ANS-water spectrum is enlarged 15 -fold.

change of either intensity or wavelength (results not shown). These data were taken to indicate that the fungal surface is responsible for the spectral shifts of ANS and that the reagent does not penetrate the interior of the cells. Similar studies were performed with all of the fungal strains cultured in the various growth media as described in Methods. All of the corresponding data dealing with the spectral properties of ANS in the presence of $C$. albicans are summarized in Table 2.

To assign a global value for the non-polar nature of the $C$. albicans surface, the measured $\lambda_{\max }$ of ANS (Table 2) was compared with the relationship between $\lambda_{\max }$ and $\varepsilon$ (Fig. 1). From this comparison of Fig. 1 with the $\lambda_{\max }$ of the ANS/C. albicans system following the various treatments, it is possible to give a ranked order of the 'equivalent' $\varepsilon$ $(\equiv \varepsilon)$ of the $C$. albicans surface as shown in Table 2 . In other words, the environment of the ANS on the fungal surface is suggested to reflect the 'ambient' hydrophobicity if it is found to possess an equivalent polarity $(\equiv \varepsilon)$ to that of a solvent of the same dielectric constant if their $\lambda_{\max }$ values are the same. On this basis, a number of trends are apparent: the YNB + Gal results in the shortest $\lambda_{\max }$ value for each strain under the various culture conditions, indicating that the YNB + Gal-cultured fungi possess the least polar or the most hydrophobic surface properties. Comparing all the strains, GDH 2346 was the most hydrophobic overall and was only matched by the other two strains when they were cultured in the $\mathrm{YNB}+\mathrm{Gal}$.

The $\lambda_{\max }$ values for the RPMI 1640- and YNB+Glccultured cells of all three $C$. albicans strains tested were within $\sim 10 \mathrm{~nm}$ of each other, whereas the YNB + Galcultured cells were $\sim 15 \mathrm{~nm}$ blue-shifted from the RPMI 1640-cultured cells of strains MRL 3153 and GRI 681. For strain GDH 2346, the YNB+Gal-cultured cells were blue-shifted by $24 \mathrm{~nm}$. These data indicate that RPMI 1640 or YNB + Glc do not cause major differences in the hydrophobic nature of the cell surface. However, the larger blue-shift exhibited by $C$. albicans, especially strain GDH 2346 cultured in YNB+Gal, shows that this medium promotes greater $\mathrm{CSH}$. The ANS fluorescence data also convey the same trend in hydrophobicity for each of the three strains. A trend also exists between each strain, GDH 2346 being most hydrophobic, followed by MRL 3153 and GRI 681.

The $\lambda_{\max }$ of ANS in the presence of each of the three strains was found to be dependent upon the culture medium (Table 2). The $\lambda_{\max }$ always appeared to be more blue-shifted for the RPMI 1640-cultured cells than the YNB + Glc-cultured cells; YNB + Gal-cultured cells were more blue-shifted than those cultured in RPMI 1640 or in YNB + Glc, which appear less hydrophobic. In terms of the fluorescence intensities of ANS observed at $\lambda_{\max }$, it is evident that a fairly similar ranked order to that found for the $\lambda_{\max }$ also results. The intensity of the fluorescence, however, is taken to indicate the relative number of

Table 2. Summary of the hydrophobic characteristics of $C$. albicans

\begin{tabular}{|c|c|c|c|c|c|}
\hline Strain & $\begin{array}{l}\text { Growth } \\
\text { medium }\end{array}$ & $\lambda_{\max }(\mathrm{nm})^{*}$ & $\equiv \varepsilon$ & $\begin{array}{l}\text { Intensity } \\
\text { at } \lambda_{\max }{ }^{*} \dagger\end{array}$ & $\varepsilon_{\mathrm{s}}^{*}$ \\
\hline \multirow[t]{3}{*}{ GDH 2346} & $\mathrm{YNB}+\mathrm{Gal}$ & $425(1)$ & $<2$ & $34(1)$ & $141 \cdot 3(3)$ \\
\hline & RPMI 1640 & $449(3)$ & $<2$ & $13 \cdot 5(2)$ & $92 \cdot 7(1)$ \\
\hline & $\mathrm{YNB}+\mathrm{Glc}$ & $455(5)$ & 2 & $13 \cdot 5(4)$ & $207 \cdot 3(7)$ \\
\hline \multirow[t]{3}{*}{ MRL 3153} & $\mathrm{YNB}+\mathrm{Gal}$ & $442(2)$ & $<2$ & $13 \cdot 5(2)$ & $136.5(2)$ \\
\hline & RPMI 1640 & $457(6)$ & 2 & $13 \cdot 5(4)$ & $163.5(4)$ \\
\hline & $\mathrm{YNB}+\mathrm{Glc}$ & $467(8)$ & 20 & $6.0(6)$ & $167 \cdot 1(5)$ \\
\hline \multirow[t]{3}{*}{ GRI 681} & $\mathrm{YNB}+\mathrm{Gal}$ & $450(4)$ & $<2$ & $13 \cdot 5(3)$ & $141 \cdot 3(3)$ \\
\hline & RPMI 1640 & $466(7)$ & 13 & $13.5(5)$ & $192 \cdot 3(6)$ \\
\hline & $\mathrm{YNB}+\mathrm{Glc}$ & $476(9)$ & 28 & $6.0(5)$ & $247 \cdot 5(8)$ \\
\hline
\end{tabular}

* Ranking on the basis of degree of CSH is given in parentheses.

† Ranking combined with wavelength data. 


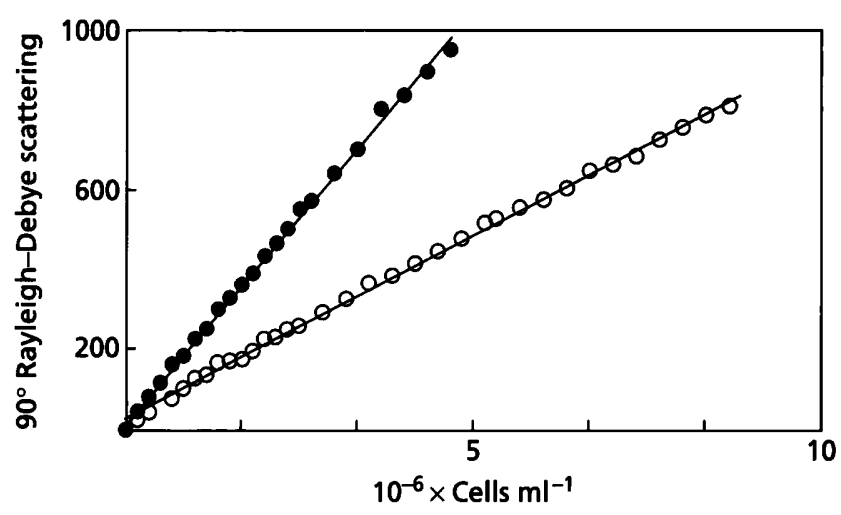

Fig. 3. Relationship between $90^{\circ}$ Rayleigh-Debye scattering at $600 \mathrm{~nm}$ following irradiation at $600 \mathrm{~nm}$ and $C$. albicans cell concentration. The data shown are for $C$. albicans strain GDH 2346 cultured in YNB + $50 \mathrm{mM}$ glucose (O) and RPMI 1640 (O), each suspended in $1 \mathrm{mM}$ HEPES, $\mathrm{pH} 7 \cdot 5$, at the indicated cell concentrations. The respective values of $\varepsilon_{\mathrm{s}}$ are $207\left(r^{2} 0.998\right)$ and 93 ( $\left.r^{2} 0.998\right)$.

molecules of ANS bound to the fungal surface once normalized by the effect of $\varepsilon$ on the intensity at a given $\lambda_{\max }$ (Fig. 1). This may then be used to indicate the relative abundance of the number of hydrophobic 'sites' present on the cell surface of each of the respective preparations of $C$. albicans, as listed in Table 2.

\section{Use of $90^{\circ}$ Rayleigh-Debye light scattering to assess the aggregation state of a suspension of $C$. albicans}

To assess the effect of CSH on self-aggregation of $C$. albicans, a $90^{\circ}$ Rayleigh-Debye light-scattering technique has been developed. The light intensity resulting from $90^{\circ}$ Rayleigh-Debye scattering by yeast cells was recorded against time whilst the concentration of yeast cells was successively increased by the serial addition of aliquots from a stock suspension. The scattering intensity increased linearly with the fungal concentration (Fig. 3). The gradient of this relationship, however, was strongly dependent upon the size of the particles; the greater the number of particles the greater the degree of scattering, but the larger the particles the smaller the scattering. Scattering measurements were performed for each strain of $C$. albicans cultured in the three different media (see Methods). In each case, as the cell number was increased the amount of scattered light also increased linearly (Fig. 3 ). The rate of the increase of the scattering intensity with respect to the cell concentration, therefore, represents a simple means of comparison of the relative ability of the yeast cells to aggregate. The numerical value of this gradient, which was defined in equation (2) as the scattering coefficient $\left(\varepsilon_{\mathrm{s}}\right)$, was determined by linear regression. $\varepsilon_{\mathrm{s}}$ was found to differ significantly and be dependent on the strain, the culture conditions and the experimental medium in which the scattering measurements were performed. An example of this variation of scattering coefficient $\left(\varepsilon_{\mathrm{s}}\right)$ is also shown in Fig. 3, and a summary of all such scattering data is presented in Table
2. $\varepsilon_{\mathrm{s}}$ may therefore be used as a gauge of the degree of aggregation of the fungal particles. Both the strain and the growth medium greatly affect this parameter in a similar manner to the ANS data. The value of $\varepsilon_{\mathrm{s}}$ obtained for the three strains of $C$. albicans generally increase as follows: GDH $2346<$ MRL $3153<$ GRI 681. Taken by itself this implies the CSH of these three strains increases in the following manner: GRI 681 < MRL 3153 < GDH 2346, which is in agreement with the conclusions of the ANS fluorimetry study (Table 2).

The nature of the growth medium also influenced the $\mathrm{CSH}$ for all three strains tested. For each strain the most hydrophobic cells were those grown in YNB+500 mM galactose and RPMI 1640 (1 M glucose). The growth medium resulting in the lowest $\mathrm{CSH}$ for all strains was YNB + $50 \mathrm{mM}$ glucose.

\section{DISCUSSION}

The spectral properties of ANS were found to vary with the dielectric constant of the solvent. Both the $\lambda_{\max }$ and the overall intensity at this wavelength vary with $\varepsilon$ in a well-defined manner (Fig. 1). The $\lambda_{\max }$ of ANS was strongly dependent on the $C$. albicans strain and the culture medium (Table 2). Fig. 1 enables the corresponding or the equivalent dielectric constant to be identified for each emission maximum for each fungal preparation (also shown in Table 2), thereby affecting the degree of $\mathrm{CSH}$. The fluorescence intensity at this emission maximum then yields the relative abundance of such 'sites' on the cell surface which presumably also contribute to the overall $\mathrm{CSH}$. The underlying mechanisms of the fluorescent processes of ANS appear to be understood (Hobden et al., 1995), and, with a few reservations, ANS would appear to function as a useful indicator of the $\mathrm{CSH}$ of the $C$. albicans. The effects of $\mathrm{CSH}$ on the ability of the $C$. albicans to self-aggregate is evident from the data shown in Table 2 derived as shown in Fig. 3. The two spectroscopic techniques outlined here for investigating the $\mathrm{CSH}$ of $C$. albicans are therefore complementary, and the results are in good agreement with work by other research groups using different techniques $(\mathrm{McC}$ courtie \& Douglas, 1981, 1984; Hazen et al., 1986; Kennedy \& Sandin, 1988; Hazen, 1990; Kennedy, 1990). McCourtie \& Douglas $(1981,1984)$ and Kennedy $(1990)$ found that the production of adhesive cells of $C$. albicans cultured in YNB was dependent upon carbohydrate supplements to the growth medium. These groups observed that cells grown in YNB+500 mM galactose were significantly more adhesive than those grown in YNB + Glc. Similarly, medium prepared for the present study yielded results from $90^{\circ}$ Rayleigh-Debye scattering and ANS spectrofluorimetry which were in good agreement with those of McCourtie \& Douglas (1981, 1984). In view of these observations it seems likely that this elevated level of adhesion may result from the greater degree of hydrophobicity exhibited by the fungus when grown under these conditions. The data in Table 2, however, indicate that both the number of such sites as well as the overall degree of hydrophobicity can be estimated by the 
techniques outlined here. In addition, it was found that the various $C$. albicans strains possessed different inherent $\mathrm{CSH}$ when cultured in the same medium but similar responses in terms of $\mathrm{CSH}$ when cultured in the different media.

Correlation of the studies of Rayleigh-Debye scattering and ANS fluorescence (Table 2) indicates that, by and large, strains exhibiting a greater $\mathrm{CSH}$ as judged by the $\lambda_{\max }$ and the fluorescence intensity of the bound ANS also aggregate to a greater extent and therefore possess a smaller $\varepsilon_{\mathrm{s}}$. It is clear, however, that this correlation is not completely unequivocal (Table 2). GDH 2346 when cultured in YNB + Gal for example, is ranked first in terms of $\mathrm{CSH}$ by ANS fluorescence (because it exhibits the shortest $\lambda_{\max }$; see Fig. 1) together with the relative abundance of such 'sites' (i.e. due to the relative fluorescence intensity), but is ranked third by $\varepsilon_{\mathrm{s}}$. These apparent idiosyncrasies may be resolved, however, by considering all the factors which are involved in aggregation. It should be emphasized that any degree of yeast-cell aggregation, as revealed by the Rayleigh-Debye scattering analysis, is an average property of the sum of all the attractive and repulsive interactions between each member of the yeast-cell population. The ANS spectrofluorimetry, however, solely reveals the $\mathrm{CSH}$. Thus, although it is clear from Table 2 that all the $C$. albicans cultures possess varying $\mathrm{CSH}$ and that this may dominate the interactions between the yeast cells (thereby lowering $\varepsilon_{\mathrm{s}}$ ), other types of physical interaction must also be considered.

These additional interactions which may not dominate the yeast-yeast interactions, but would appear to modulate them, are thought to be electrostatic in origin, and we have suggested that such interactions between yeasts of $C$. albicans are invariably repulsive (Jones \& O'Shea, 1994). The discrepancy between the estimated value of $\varepsilon_{\mathrm{s}}$ for galactose-cultured GDH 2346 ranked third in terms of the overall aggregation state but first in terms of $\mathrm{CSH}$ as judged by the ANS fluorescence, when compared to the first-ranked $\varepsilon_{\mathrm{s}}$ value of GDH 2346 cultured in RPMI 1640 but ranked third in terms of the observed $\mathrm{CSH}$, may be resolved in the light of the influence of these additional interactions. Studies of the electrostatic nature of these respective cultures indicate that GDH 2346 cultured in $\mathrm{YNB}+\mathrm{Gal}$ has a greater electronegativity than when cultured in RPMI 1640 (unpublished observations). This means that YNB + Gal-cultured GDH 2346, which appear to be more hydrophobic than the RPMI 1640-cultured cells (Table 2), must also exhibit more electrostatic repulsion between the yeast cells. The balance of attractive and repulsive forces, therefore, appears to be less in favour of aggregation for the galactose culture of GDH 2346 than the RPMI 1640 culture.

The use of hydrophobic beads to determine CSH has been comprehensively discussed by Hazen (1990), who points out the advantage and disadvantages of this technique. Several advantages of this technique are common to the techniques outlined in the present paper, for example no modification of the fungal surface by solvents or other reagents is necessary. The hydrophobic-particle-attachment assay suffers slightly from problems of quantitative assessments of the degree of $\mathrm{CSH}$, i.e. it is not easy to discriminate between the degree of hydrophobicity of a putative 'site' and the number of such sites and the resolution is limited to some extent by the size of the particles. The present techniques offer information of this nature, but their shortcoming is that they do not yield information of the spatial disposition of such 'sites', whereas the hydrophobic bead assays do yield such information. We are currently using confocal fluorescence microscopy to identify the spatial variation (if any) of the number and degree of hydrophobic sites on the cell surface, and this is being compared with the information from hydrophobic-bead-binding assays.

The nature of fungal CSH is important and a number of methods for its study are now available (Hazen, 1990); the techniques outlined in this paper should add to the repertoire of established methods. These methods, in combination with a related technique which deals exclusively with the electrostatic properties of the fungal cell surface, as outlined by Jones et al. (1995), mean that in principle, it is possible to characterize independently all the physical properties of the $C$. albicans surface which are involved in the interaction between the fungi and other surfaces under a large number of circumstances. Such techniques may also be suitable for use with other microorganisms.

\section{REFERENCES}

Calderone, R. A. \& Braun, P. (1991). Adherence and receptor relationships of Candida albicans. Microbiol Rev 55, 1-10.

Critchley, I. A. \& Douglas, L. J. (1987). Isolation and partial characterization of an adhesin from Candida albicans. J Gen Microbiol 133, 629-636.

Douglas, L. J. (1985). Adhesion of pathogenic Candida species to host surfaces. Microbiol Sci 2, 243-247.

Hazen, K. C. (1989). Participation of yeast cell surface hydrophobicity in adhesion of Candida albicans to human epithelial cells. Infect Immun 57, 1984-1900.

Hazen, K. C. (1990). In Microbial Cell Surface Hydropbobicity, pp. 249-295. Edited by R. J. Doyle \& M. Rosenberg. Washington, DC: American Society for Microbiology.

Hazen, K. C. \& Hazen, B. W. (1987). Temperature-modulated physiological characteristics of Candida albicans. Microbiol Immunol 31, 497-508.

Hazen, K. C. \& Hazen, B. W. (1988). Dynamic expression of cell surface hydrophobicity during initial yeast cell growth and before germ tube formation of Candida albicans. Infect Immun 56, 2521-2525.

Hazen, K. C., Plotkin, B. J. \& Klimas, D. M. (1986). Influence of growth conditions on cell surface hydrophobicity of $C$ andida albicans and Candida glabrata. Infect Immun 54, 269-271.

Hobden, C., Ayoub, F., Wall, J. \& O'Shea, P. (1995). The interaction of arylnaphthalenesulfonates with albumin. (in press).

Jones, L. \& O'Shea, P. (1994). The electrostatic nature of the cell surface of Candida albicans: a role in adhesion. Exp Mycol 18, 1-10.

Jones, L., Hobden, C. \& O'Shea, P. (1995). The use of a real-time probe of the electrostatic properties of the cell surface of Candida albicans. Mycol Res 99, 969-976. 
Kaeppeli, O., Mueller, M. \& Feichter, A. (1978). Chemical and structural alterations at the cell surface of Candida tropicalis, induced by hydrocarbon substrate. $J$ Bacteriol 133, 952-958.

Kennedy, M. J. (1990). Models for studying the role of fungal attachment in colonization and pathogenesis. Mycopatbologia 109, 123-137.

Kennedy, M. J. \& Sandin, R. L. (1988). Influence of growth conditions on Candida albicans adhesion, hydrophobicity and cell wall ultrastructure. J Med Vet Mycol 26, 79-92.

Kerker, M. (1969). The Scattering of Light and Other Electromagnetic Radiation. New York: Academic Press.

Klotz, S. A. (1989). Surface-active properties of Candida albicans. Appl Environ Microbiol 55, 2119-2122.

Klotz, S. A. \& Penn, R. L. (1987). Multiple mechanisms may contribute to the adhesion of Candida yeasts to living cells. Curr Microbiol 16, 119-122.

Klotz, S. A., Drutz, D. J. \& Zajic, J. E. (1985). Factors governing adhesion of Candida species to plastic surfaces. Infect Immun 50, 97-101.

Lachica, R. V. \& Zink, D. L. (1984). Determination of plasmidassociated hydrophobicity of Yersinia enterocolitica by a latex particle agglutination test. J Clin Microbiol 19, 660-663.

Latimer, P. (1979). Light scattering vs. microscopy for measuring average cell size and volume. Biophys $J$ 27, 117-126.

Lindahl, M., Faris, A., Wadstrom, T. \& Hjerten, S. (1981). A new test based on 'salting out' to measure relative surface hydrophobicity of bacterial cells. Biochim Biophys Acta 677, 471-476.

Meunier, F. (1989). Candidiasis. Eur J Clin Microbiol Infect Dis 8, 438-447.

McCourtie, J. \& Douglas, L. J. (1981). Relationship between cell surface composition of Candida albicans and adhesion to acrylic after growth on different carbon sources. Infect Immun 32, 1234-1241.

McCourtie, J. \& Douglas, L. J. (1984). Relationship between cell surface composition, adhesion and virulence of Candida albicans. Infect Immun 45, 6-12.
Minagi, S., Miyake, Y., Fujioka, Y., Tsuru, H. \& Suginaka, H. (1986). Cell-surface hydrophobicity of Candida species as determined by the contact-angle and hydrocarbon-adherence methods. $J$ Gen Microbiol 132, 1111-1115.

O'Shea, P. (1991). The role of electrostatic and electrodynamic forces in fungal morphogenesis and host infection. In Fungal Cell Wall and Immune Response, pp. 285-302, NATO ASI series vol. H53. Edited by J. P. Latge \& D. Boucias. Springer-Verlag: Heidelberg. Pines, O., Shoham, Y., Rosenberg, E. \& Gutnick, D. (1988). Unmasking of surface components by removal of cell-associated emulsan from Acinetobacter sp. RAG-1. Appl Microbiol Biołechnol 28, 93-99.

Reinhart, H., Muller, G. \& Sobel, J. D. (1985). Specificity and mechanism of in vitro adhesion of Candida albicans. Ann Clin Lab Sci 15, 406-413.

Rosenberg, M. \& Kjelleberg, S. (1986). Hydrophobic interactions: role in bacterial adhesion. Adv Microb Ecol 9, 353-393.

Rosenberg, M., Gutnick, D. \& Rosenberg, E. (1980). Adhesion of bacteria to hydrocarbons: a simple method for measuring cellsurface hydrophobicity. FEMS Microbiol Lett 9, 29-33.

Rotrosen, D., Gibson, T. R. \& Edwards, J. E., Jr (1983). Adhesion of Candida species to intravenous catheters. J Infect Dis 147, 594-595.

Shepherd, M. G. (1987). Cell envelope of Candida albicans. Crit Rev Microbiol 15, 7-25.

Slavik, J. (1982). Anilinonaphthalene sulfonic acid as a probe of membrane composition and function. Biocbim Biopbys Acta 694, 1-25.

Smyth, C. J., Jonsson, P., Olsson, E., Soderlind, O., Rosengren, J., Hjerten, S. \& Wadstrom, T. (1978). Differences in hydrophobic surface characteristics of porcine enteropathogenic Escherichia coli with or without $\mathrm{K} 88$ antigen as revealed by hydrophobic interaction chromatography. Infect Immun 22, 462-472.

Received 16 January 1995; revised 16 March 1995; accepted 31 March 1995. 